\title{
Quantization on the Virasoro Group
}

\author{
Victor Aldaya $^{1, \star}$ and José Navarro-Salas ${ }^{2}$ \\ ${ }^{1}$ The Blackett Laboratory, Imperial College, London SW7 2BZ, UK \\ ${ }^{2}$ Departamento de Fisica Teórica, Facultad de Fisicas, Universidad de Valencia, Burjasot 46100 - \\ Valencia, Spain, and IFIC (Centro Mixto Universidad de Valencia-C.S.I.C.), Spain
}

\begin{abstract}
The quantization of the Virasoro group is carried out by means of a previously established group approach to quantization. We explicitly work out the two-cocycles on the Virasoro group as a preliminary step. In our scheme the carrier space for all the Virasoro representations is made out of polarized functions on the group manifold. It is proved that this space does not contain null vector states, even for $c \leqq 1$, although it is not irreducible. The full reduction is achieved in a straightforward way by just taking a well defined invariant subspace $\mathscr{H}_{(c, h)}$, the orbit of the enveloping algebra through the vacuum, which is irreducible for any value of $c$ and $h . \mathscr{H}_{(c, h)}$ is a proper subspace of the space of polarized functions for those values of $c$ and $h$ for which the Kac determinant is zero. We give the local version of these group representations as well as the associated classical phase space structures, i.e., symplectic form and Noether invariants.
\end{abstract}

\section{Introduction}

The Geometric Quantization of Co-adjoint Orbits of a Lie group $G[1,2]$ is intended to be the quantum mechanical description of a dynamical system defined by the group $G$ itself. In GQCO the phase space $M$ of the dynamical system is constituted by the symplectic manifold structure with which the coadjoint orbits are naturally endowed. The Hilbert space of wave functions is a space of sections of a line bundle on the symplectic manifold $M$ once the so-called polarization (or Planck) conditions are imposed [1]. This scheme aims to establish in this way a correspondence between the set of irreducible unitary representations (quantizations) of $G$ and that of its co-adjoint orbits. Nevertheless this association is in general not quite well achieved, as has been made evident when applied to the

Research partially supported by the Conselleria de Cultura de la Generalitat Valenciana, the Plan de formación del Personal investigador, the Comision Interministerial de Ciencia y Tecnologia (CICYT) and the British Council

^ On leave of absence from 2 
Virasoro group [2]. The underlying philosophy of GQCO, i.e., the description of the quantum behavior of physical systems from (or on) a group manifold, is the base of several group theoretic formulations [3-12].

In this paper we undertake the task of quantizing the Virasoro group through a Group Approach to Quantization (GAQ) $[5,10,11]$ whose starting point is an entire group manifold rather than a symplectic manifold (co-adjoint orbit). The basic structure of the GAQ formalism is a "quantum group" $\widetilde{G}$, i.e., a Lie group endowed with a principal bundle structure with fiber a one dimensional subgroup $T(U(1)$ or $\mathbb{R})$ [5]. (See ref. [12] for a generalization in which $T$ is replaced by a nonabelian, non-compact subgroup.) Typical finite-dimensional quantum groups are the centrally extended Galilei group [5] (see ref. [13]), the Poincare group [6] $S U(2)[10], S L(2, \mathbb{R})[14]$ (see ref. [15]) and the infinite-dimensional case: the KacMoody groups [10,11] (see ref. [16]) and the subject of the present paper, the Virasoro group.

As in the finite-dimensional case the $T$-fibration $\operatorname{diff} S^{1} \rightarrow \operatorname{diff} S^{1}$ associated with the non-trivial central extension of $\operatorname{diff} S^{1}$ (as well as a pseudo-extension or trivial extension closely related to the fibration $\operatorname{diff} S^{1} \rightarrow \operatorname{diff} S^{1} /\left\langle l_{0}\right.$-subgroup $\rangle$, see later on) allows us to single a vertical component $\Theta[5,6,10]$ out of the leftinvariant canonical 1 -form [17]. This globally defined 1 -form is a connection form and can be locally calculated as the left-invariant 1-form dual to the fundamental vector field (central generator). On the other hand $\Theta$ is a pre-contact 1-form on diff $S^{1}$ whose characteristic module $C_{\Theta} \equiv\left\langle\tilde{X} \in X\left(\right.\right.$ diff $\left.\left.S^{1}\right) / i_{\tilde{X}} \Theta=0=i_{X} d \Theta\right\rangle$ is generated by a subalgebra $\mathscr{G}_{\Theta}$, the characteristic subalgebra, of left-invariant vector fields in the kernel of the Lie algebra cocycle. Indeed a quantum manifold in the ordinary sense [1] may be obtained from $\left(\operatorname{diff} S^{1}, \Theta\right.$ ) by taking the quotient by $\mathscr{G}_{\Theta}$ and further quotient by $T$ would lead to the classical symplectic manifold $\left(\right.$ diff $\left.\widetilde{S} S^{1}, d \Theta\right) /\left(\mathscr{G}_{\Theta} \oplus T\right)$. The quantum representations of the Virasoro group will be obtained from those $T$-equivariant functions on the group, $\Psi \in \mathscr{F}\left(\operatorname{diff} S^{1}, \mathbb{C}\right)$, satisfying the polarization conditions (see Sect. IV).

We show in this paper that the space generated by the polarized functions on the group is not, in general, an orbit through the vacuum of the enveloping algebra and, therefore, it is not properly a highest weight nor irreducible module. We nevertheless prove the nice property that no null vector state is present, i.e., the vacuum is the only wave function annihilated by the annihilation operators, so that the irreducible module is obtained by simply considering the mentioned orbit through the vacuum. The concrete values of $c$ and $h$ (central charge and expectation value of the $\hat{L}_{0}$ operator respectively) for which the initial space of polarized functions is not a highest weight module, then provide the values corresponding to the existence of null vectors in the standard Virasoro Verma modules [16].

The non-irreducibility of the space of polarized functions is not a property of the infinite dimension. In the case of $\operatorname{SL}(2, \mathbb{R})$ it will be shown (see the appendix) how this space is not irreducible for $N \leqq-1$ (is not an orbit through the vacuum). This fact has not been sufficiently remarked probably because, unlike the Virasoro case, these representations are not unitary.

In order to perform the GAQ for the particular case of the Virasoro group we first need a local group law near the identity from which explicit calculations can 
be achieved. Thus, in Sect. II we give an exact formal group law, including the twococycle, obtained by using a "perturbative" method specially designed for those purposes [10]. In Sect. III we study the different characteristic moduli of the quantization 1-form $\Theta$ according to the possible values of $h$ and $c$. In Sect. IV the Virasoro quantization is accomplished. We work out the (polarized) wave functions, discuss the above mentioned problem concerning the irreducibility and construct, explicitly, the Lie algebra representations from those of the group. Finally in Sect. V our approach is compared with the coadjoint orbits method. The paper is supplemented by an appendix where the GAQ is briefly illustrated on the $S L(2, \mathbb{R})$ subgroup of the Virasoro group.

\section{Formal Group Law for diff $\boldsymbol{S}^{\mathbf{1}}$. Two-Cocycle for the Virasoro Group}

In order to achieve explicit calculations on the Virasoro group we start looking for a formal group law [18] up to the $\infty$ order of the centrally extended group diff $S^{1}$. That means finding an exact solution for the formal power series for the parameters of the unextended group,

$$
\begin{aligned}
l_{m}^{\prime \prime}= & l_{m}^{\prime}+l_{m}+B_{i, j}^{m} l_{i}^{\prime} l_{j}+\frac{1}{2 !} B_{i j, k}^{m} l_{i}^{\prime} l_{j}^{\prime} l_{k}+\frac{1}{2 !} B_{i, j}^{m} l_{i}^{\prime} l_{j} l_{k} \\
& +\frac{1}{3 !} B_{i j k, n}^{m} l_{i}^{\prime} l_{j}^{\prime} l_{k}^{\prime} l_{n}+\frac{1}{2 !} \frac{1}{2 !} B_{i j, k n}^{m} l_{i}^{\prime} l_{j}^{\prime} l_{k} l_{n}+\ldots
\end{aligned}
$$

as well as another for the central parameter

$$
\varphi^{\prime \prime}=\varphi^{\prime}+\varphi+B_{i, j}^{\varphi} l_{i}^{\prime} l_{j}+\frac{1}{2 !} B_{i j, k}^{\varphi} l_{i}^{\prime} l_{j}^{\prime} l_{k}+\frac{1}{2 !} B_{i, j k}^{\varphi} l_{i}^{\prime} l_{j} l_{k}+\ldots
$$

where

$$
\begin{aligned}
& B_{i, j}^{m}-B_{j, i}^{m} \equiv C_{i j}^{m}=\sqrt{-1}(j-i) \delta_{m, i+j}, \\
& B_{i, j}^{\varphi}-B_{j, i}^{\varphi} \equiv C_{i, j}^{\varphi}=\sqrt{-1} \frac{c}{12} j^{3} \delta_{j,-i}
\end{aligned}
$$

are the structure constants of the extended Lie algebra,

$$
\left[L_{n}, L_{m}\right]=-\sqrt{-1}(n-m) L_{n+m}-\frac{\sqrt{-1}}{12} c n^{3} \delta_{n,-m}
$$

and the constants $B_{i_{1} \ldots i_{k}, j_{1} \ldots j_{r}}$, which are symmetric in $\left(i_{1} \ldots i_{k}\right)$ and $\left(j_{1} \ldots j_{r}\right)$ respectively, are constrained by the associativity condition,

$$
\left(\tilde{g}^{\prime \prime} * \tilde{g}^{\prime}\right) * \tilde{g}=\tilde{g}^{\prime \prime} *\left(\tilde{g}^{\prime} * \tilde{g}\right), \quad \tilde{g}^{\prime \prime}, \tilde{g}^{\prime}, \tilde{g} \in \operatorname{dif\tilde {f}} S^{1} .
$$

The trivial two-cocycle $-1 / 12 c^{\prime} n \delta_{n,-m}$, to be added to (2.4), will be introduced later on.

A general method for calculating formal group laws from structure constants has been shown in ref. [10]. The associativity condition entails a set of constraints on the coefficients $B$ order by order whose general expressions are given by 
formulas (2.4-6) of ref. [10]. These equations do not have a unique solution but a "gauge" can be fixed which eliminates this freedom and unambiguously determines all the $B$ 's to any order. Of course, the difficulty lies in fining a valid gauge to any order, i.e., compatible with all the constraints.

In the first place we will obtain one explicit formal group law for the unextended diff $S^{1}$ group. Our gauge is

$$
\begin{gathered}
B_{i, j}^{m}=\sqrt{-1} j \delta_{m, i+j}, \\
B_{i_{1} \ldots i_{k}, j_{1} \ldots j_{r}}^{m}=0, \quad r \geqq 2 .
\end{gathered}
$$

It can then be proved that the resulting algebraic system on $B$ after imposing (2.5) is compatible and has a unique solution which displays the correct symmetry in $\left(i_{1} \ldots i_{k}\right)$,

$$
B_{i_{1} \ldots i_{k}, j}^{m}=(\sqrt{-1} j)^{k} \delta_{m, i_{1}+\ldots+i_{k}+j} .
$$

The formal group law for $\operatorname{diff} S^{1}$ thus follows:

$$
\begin{aligned}
l_{m}^{\prime \prime}= & l_{m}^{\prime}+l_{m}+\sqrt{-1} p l_{p} l_{m-p}^{\prime}+\frac{(\sqrt{-1} p)^{2}}{2 !} l_{p} l_{n}^{\prime} l_{m-n-p}^{\prime}+\ldots \\
& +\sum_{n_{1}+n_{2}+\ldots+n_{r}+p=m} \frac{(\sqrt{-1} p)^{r}}{r !} l_{p} l_{n_{1}}^{\prime} l_{n_{2}}^{\prime} \ldots l_{n_{r}}^{\prime}+\ldots
\end{aligned}
$$

Notice that the coordinates $l_{m}$ in (2.7) do not correspond to those associated with the exponential map, nor (2.7) itself is the Baker-Campbell-Hausdorff $(\mathrm{BCH})$ formula, since $l_{m}^{\prime \prime}\left(l_{n}^{\prime}=l_{n}, l_{k}\right) \neq 2 l_{m}$. The BCH formula that can be obtained through an adequate gauge, has the drawback that it does not apply to those elements out of reach of the exponential map. Yet for finite-dimensional (non-compact) connected groups one may have some group elements which do not belong to any one-parameter subgroup, as for instance, $\operatorname{diag}\left(-|\lambda|,-|\lambda|^{-1}\right)$ in $S L(2, \mathbb{R})$ with real $\lambda$ different from 1. However, the formal group law corresponding with the power expansion of the $S L(2, \mathbb{R})$ composition law (A.1) is rid of this difficulty.

The actual meaning of the local coordinates $l_{m}$ in our particular choice of the gauge might be established by means of the Noether invariants which could be compared with those given in terms of the coordinates for which the diffeomorphism $\theta \rightarrow S(\theta)$ is written as $\theta+1 / 2 \pi \Sigma s_{n} \exp -i n \theta$. They are found in the first reference in ref. [2] although the computation is given to the lowest order.

We now calculate the central group law (2.2) which may be written as

$$
\varphi^{\prime \prime}=\varphi^{\prime}+\varphi+\xi\left(g^{\prime}, g\right), \quad g^{\prime}, g \in \operatorname{diff} S^{1},
$$

thus defining a two-cocycle $\xi$ on diff $S^{1}$. The gauge to be chosen must be more involved, as it has to be compatible with the Lie algebra cocycle (2.3b) as well as the formal group law for diff $S^{1}$ just found (2.7) and has to lead to $B$ coefficients, $B_{i_{1} \ldots i_{k}, j_{1} \ldots j_{r}}$, with the proper index symmetry. Our guess for the gauge is

$$
\begin{gathered}
B_{i, j}^{\varphi}=\frac{\sqrt{-1} c}{24} j i^{2} \delta_{i,-j}, \\
B_{i, j_{1} \ldots j_{r}}^{\varphi}=\frac{(\sqrt{-1})^{r} c}{24}(-)^{r+1}(r-1) ! j_{1} \ldots j_{r} i^{2} \delta_{i,-j_{1}-\ldots-j_{r}} .
\end{gathered}
$$


The resulting system of constraint equations is also compatible once (2.8) are taken into account and has a unique solution. The general formula giving the $B^{\varphi}$ 's to arbitrary order is

$$
\begin{aligned}
B_{i_{1} \ldots i_{k}, j_{1} \ldots j_{r}}^{\varphi}= & \frac{(\sqrt{-1})^{k+r-1}}{24}(-)^{r+k}(r-1) ! j_{1} \ldots j_{r} \\
& \times\left(i_{1}+i_{2}+\ldots+i_{k}\right) P^{(k)}\left(i_{1}, \ldots, i_{k}\right) \delta_{i_{1}+\ldots+i_{k}+j_{1}+\ldots+j_{r}, 0}
\end{aligned}
$$

In (2.9) $P^{(k)}\left(i_{1}, \ldots, i_{k}\right)$ is a symmetric homogeneous polynomial of degree $k$ in the variables $i_{1}, \ldots, i_{k}$,

$$
P^{(k)}\left(i_{1}, \ldots, i_{k}\right)=\sum_{\operatorname{part}(k)} a_{\lambda_{1} \ldots \lambda_{s}}^{(k)} i_{1}^{\lambda_{1}} \ldots i_{s}^{\lambda_{s}},
$$

where the sum goes over the partitions $\left[\lambda_{1}, \ldots, \lambda_{s}\right]$ of $k$. The coefficients, $a_{\lambda_{1} \ldots \lambda_{s}}^{(k)}$, are recursively given as

$$
a_{\lambda_{1} \ldots \lambda_{s}}^{(k)}=\left\{\begin{array}{l}
(k-1) ! \text { if } \quad\left[\lambda_{1}, \ldots, \lambda_{s}\right]=[1, \ldots, 1] . \\
\sum_{i=1}^{k} a_{\lambda_{1} \ldots \lambda_{i}-1 \ldots \lambda_{s}}^{(k-1)}
\end{array}\right.
$$

Thus, for instance, the lowest polynomials $P^{(k)}$ are

$$
\begin{aligned}
P^{(1)}(x)= & x, \quad P^{(2)}(x, y)=x^{2}+y^{2}+x y, \\
P^{(3)}(x, y, z)= & x^{3}+y^{3}+z^{3}+2\left(x^{2} y+x^{2} z+y^{2} x+y^{2} z\right. \\
& \left.+z^{2} x+z^{2} y\right)+2 x y z, \\
P^{(4)}(x, y, z, u)= & x^{4}+\ldots+u^{4}+3\left(x^{2} y+\ldots\right)+6\left(x^{2} y z+\ldots\right) \\
& +4\left(x^{2} y^{2}+\ldots\right)+6 x y z u .
\end{aligned}
$$

The two-cocycle $\xi$ is derived from the $\varphi$ group law $\left(\xi=\varphi^{\prime \prime}-\varphi^{\prime}-\varphi\right)$,

$$
\begin{aligned}
\varphi^{\prime \prime}=\varphi^{\prime}+\varphi+\xi\left(g^{\prime}, g\right)= & \varphi^{\prime}+\varphi-\frac{c}{24}\left[(-\sqrt{-1})(-n) n^{2} l_{-n} l_{n}^{\prime}\right. \\
& +\frac{(-\sqrt{-1})^{2}}{2 !} n_{1} n_{2}\left(n_{1}+n_{2}\right)^{2} l_{n_{1}} l_{n_{2}} l_{-n_{1}-n_{2}}^{\prime} \\
& -\frac{(-\sqrt{-1})^{2}}{2 !}\left(n_{1}+n_{2}\right)^{2}\left(n_{1}^{2}+n_{2}^{2}+n_{1} n_{2}\right) \\
& \times l_{-n_{1}-n_{2}} l_{n_{1}}^{\prime} l_{n_{2}}+\ldots \\
& +\frac{(-\sqrt{-1})^{k+r-1}}{k ! r !}(r-1) ! m_{1} \ldots m_{r}\left(n_{1}+\ldots+n_{k}\right) \\
& \left.\times P^{(k)}\left(n_{1}, \ldots, n_{k}\right) l_{m_{1}} \ldots l_{m_{r}} l_{n_{1}}^{\prime} \ldots l_{n_{k}}^{\prime}+\ldots\right],
\end{aligned}
$$

which along with (2.7) constitute the formal group law for the centrally extended Virasoro group diff $S^{1}$. 
At this point it must be remarked that the unextended group $\operatorname{diff} S^{1}$ already undergoes a non-trivial fibration $\operatorname{diff} S^{1} \rightarrow \operatorname{diff} S^{1} /\left\langle l_{0}\right\rangle$ conferring on it the structure of a quantum group. This constitutes a natural generalization of the three fibrations of the subgroup $S L(2, \mathbb{R})$ (parametrized by $l_{0}, l_{ \pm 1}$ ): one $U(1)$-fibration on a two-sheeted hyperboloid and two $\mathbb{R}$-fibrations on a one-sheeted hyperboloid and a (light-)cone respectively. Nevertheless we shall always call $l_{0}$ the parameter of the structure group irrespective of its topology rather than denominating $l_{0}$ the $U(1)$ parameter and $l_{ \pm 1}$ the other non-compact parameters. The advantage in so doing is the possibility of handling all cases together. We must, however, be aware of the actual topology of the fibration in question when doing the local calculations.

The simplest way of incorporating the " $l_{0}$ "-fibrations into the main fibration $\operatorname{diff} S^{1} \rightarrow \operatorname{diff} S^{1}$ is by means of the pseudo-extension concept $[6,10]$. The non-trivial $l_{0}$-fibration $\operatorname{diff} S^{1} \rightarrow \operatorname{diff} S^{1} /\left\langle l_{0}\right\rangle$ can equally be treated as a "trivial" central extension of diff $S^{1}, \operatorname{diff} S^{1} \widetilde{\otimes}\left\langle l_{0}\right\rangle \rightarrow \operatorname{diff} S^{1}$, achieved by means of a (pseudo-)cocycle (coboundary actually) locally generated by a linear function on $l_{0}$. This class of coboundaries, which indeed modify the structure constants of the Lie algebra define pseudo-extended groups looking like non-trivial extension under the GAQ formalism $(d \Theta \neq 0)$. The group law for the parameter of the $U(1)$ central subgroup in $\operatorname{diff} S^{1} \tilde{\otimes}\left\langle l_{0}\right\rangle$ is $\left[\zeta \in U(1)\right.$, locally $\left.\zeta=e^{i \varphi}\right]$

$$
\zeta^{\prime \prime}=\zeta^{\prime} \zeta e^{-i c^{\prime}\left(l_{0}^{\prime \prime}-l_{0}^{\prime}-l_{0}\right)}
$$

and thus, a real number $c^{\prime}$ comes into the theory. This number must be an integer if $l_{0}$ parametrizes the $U(1)$ subgroup of $\operatorname{diff} S^{1}$. In that case we are generalizing the discrete $S L(2, \mathbb{R})$ representations associated with the $U(1)$-fibration on the twosheeted hyperboloid (see appendix). If we wanted to represent just the Lie algebra we would consider continuous values of $c^{\prime}$ irrespective of the topology of the $l_{0}$-subgroup. We finally propose for diff $S^{1}$ the group law (2.7) in addition to the following one for the $U(1)$ central parameter:

$$
\begin{gathered}
\varphi^{\prime \prime}=\varphi^{\prime}+\varphi+\frac{c}{24} \xi\left(g^{\prime}, g\right)-\frac{c^{\prime}}{24} \xi_{\mathrm{cob}}\left(g^{\prime}, g\right), \\
\xi_{\mathrm{cob}}\left(g^{\prime}, g\right) \equiv l_{0}^{\prime \prime}-l_{0}^{\prime}-l_{0},
\end{gathered}
$$

where $\xi\left(g^{\prime}, g\right)$ is read from (2.13) and $l_{0}^{\prime \prime}$ from (2.7).

To end this section, and for the sake of completeness we include different actions of the Virasoro group. We provide the vector realization corresponding to the action on the anti-ghost operators $\Psi_{n}$ of string theory, the dual one on ghost operators $\Psi^{* n}$ and the action on the string modes $\alpha_{n}^{\mu}$. According to the infinitesimal actions

$$
\begin{aligned}
{\left[X_{l_{n}}, X_{\psi_{m}}\right] } & =\sqrt{-1}(n-m) X_{\psi_{n+m}}, \\
{\left[X_{l_{n}}, X_{\psi^{* m}}\right] } & =-\sqrt{-1}(2 n-m) X_{\psi^{* m-n}}, \\
{\left[X_{l_{n}}, X_{\alpha_{m}^{\mu}}\right] } & =\sqrt{-1} m X_{\alpha_{n+m}^{\mu}},
\end{aligned}
$$


the finite actions on the field (group) variables, $\Psi_{n}, \Psi^{* m}, \alpha_{p}^{\mu}$, are derived much in the same way the action of the Virasoro group on itself was. They are

$$
\psi_{m}^{\prime}=R_{m}^{\cdot n} \psi_{n}, \quad \psi^{\prime * m}=R_{n}^{-1 \cdot m} \psi^{* n}, \quad \alpha_{m}^{\prime \mu}=U_{m}^{\cdot n} \alpha_{n}^{\mu},
$$

where

$$
\begin{aligned}
& R_{k}^{\cdot m}=\delta_{k}^{m}+\sum_{n_{1}+m=k} B_{n_{1}, m}^{k} l_{n_{1}}+\ldots+\frac{1}{j !} \sum_{n_{1}+\ldots+n_{j}+m=k} B_{n_{1} \ldots n_{j}, m}^{k} l_{n_{1}} \ldots l_{n_{j}}+\ldots, \\
& B_{n_{1}, m}^{k}=\sqrt{-1}\left(-n_{1}+m\right), \\
& B_{n_{1} n_{2}, m}^{k}=(\sqrt{-1})^{2}\left(2 n_{1} n_{2}-m\left(n_{1}+n_{2}\right)+m^{2}\right) \text {, } \\
& \stackrel{\because:}{B_{n_{1} \ldots n_{j}, m}^{k}}=(\sqrt{-1})^{j}\left[(-)^{j} j ! n_{1} n_{2} \ldots n_{j}+(-)^{j-1}(j-1) !\right. \\
& \left.\times\left(n_{1} n_{2} \ldots n_{j-1}+\ldots\right) m+\ldots+m^{j}\right], \\
& \left(R^{-1}\right)_{k}^{\cdot m}=\delta_{k}^{m}+\sum_{n_{1}+m=k} \hat{B}_{n_{1}, m}^{k} l_{n_{1}}+\ldots+\frac{1}{j !} \sum_{n_{1}+\ldots+n_{j}+m=k} \hat{B}_{n_{1} \ldots n_{j}, m}^{k} l_{n_{1}} \ldots l_{n_{j}}+\ldots, \\
& \hat{B}_{n_{1}, m}^{k}=\sqrt{-1}\left(2 n_{1}-k\right), \\
& \hat{B}_{n_{1} n_{2}, m}^{k}=(\sqrt{-1})^{2}\left(2 n_{1} n_{2}-2 k\left(n_{1}+n_{2}\right)+k^{2}\right) \text {, } \\
& {\stackrel{:}{\hat{B}_{n_{1} \ldots n_{j}, m}^{k}}}^{k}=(\sqrt{-1})^{j}\left[2 ( - k ) ^ { j - 2 } \left(n_{1} n_{2}+n_{1} n_{3}+\ldots+n_{1} n_{j}\right.\right. \\
& \left.+n_{2} n_{3}+\ldots+n_{2} n_{j}+\ldots+n_{j-1} n_{j}\right) \\
& \left.+2(-k)^{j-1}\left(n_{1}+\ldots+n_{j}\right)+(-k)^{j}\right] \text {, } \\
& U_{k}^{\cdot m}=\delta_{k}^{m}-i m l_{k-m}+\sum_{n_{1}+n_{2}=k-m} \frac{(-i m)^{2}}{2 !} l_{n_{1}} l_{n_{2}}+\ldots \\
& +\sum_{n_{1}+\ldots+n_{j}=k-m} \frac{(-i m)^{j}}{j !} l_{n_{1}} \ldots l_{n_{j}}+\ldots .
\end{aligned}
$$

\section{Quantization Form and Noether Invariants}

From (2.7), (2.15) the vertical component $\Theta$ (quantization form) of the leftinvariant canonical 1-form $\vartheta^{L}$ can be derived by duality with respect to the leftinvariant vector fields $\mathscr{X}^{L}\left(\operatorname{diff} S^{1}\right)$ :

$$
\Theta\left(\Xi \equiv \tilde{X}_{\zeta}^{L}\right)=1, \quad \Theta(\text { any other })=0 .
$$

The fields in $\mathscr{X}^{L}\left(\right.$ diff $\left.S^{1}\right)$ are easily obtained $\left(\tilde{X}^{L}=\left.\frac{\partial \tilde{g}^{\prime \prime}}{\partial \tilde{g}}\right|_{\tilde{g}=e}, \tilde{g}^{\prime \prime}=\tilde{g}^{\prime} * \tilde{g}\right)$ : 


$$
\begin{aligned}
& \tilde{X}_{l_{k}}^{L}= \frac{\partial}{\partial l_{k}}+i k l_{m-k} \frac{\partial}{\partial l_{m}}+\frac{(i k)^{2}}{2 !} l_{n} l_{m-n-k} \frac{\partial}{\partial l_{m}}+\ldots \\
&+\frac{(i k)^{j}}{j !} \sum_{n_{1}+\ldots+n_{j}=m-k} l_{n_{1}} \ldots l_{n_{j}} \frac{\partial}{\partial l_{m}}+\ldots \\
&+\frac{c}{24}\left\{(-i) k^{2}(-k) l_{-k}+\frac{(-i)^{2}}{2 !} k^{2} \sum_{n_{1}+n_{2}=-k}\left(n_{1}^{2}+n_{2}^{2}+n_{1} n_{2}\right) l_{n_{1}} l_{n_{2}}+\ldots\right. \\
&\left.+\frac{(-i)^{j}}{j !} k^{2} \sum_{n_{1}+\ldots+n_{j}=-k} P^{(j)}\left(n_{1}, \ldots, n_{j}\right) l_{n_{1}} \ldots l_{n_{j}}+\ldots\right\} \Xi \\
&-\frac{c^{\prime}}{24}\left\{i k l_{-k}+\frac{(i k)^{2}}{2 !} l_{n_{1}} l_{-k-n_{1}}+\ldots+\frac{(i k)^{j}}{j !} \sum_{n_{1}+\ldots+n_{j}=-k} l_{n_{1}} \ldots l_{n_{j}}+\ldots\right\} \Xi, \\
& \tilde{X}_{\zeta}^{L}=i \zeta \frac{\partial}{\partial \zeta} \equiv \Xi
\end{aligned}
$$

where $\sqrt{-1}$ has been converted to the customary notation. They close on the algebra $\tilde{\mathscr{G}}$ :

$$
\left[\tilde{X}_{l_{n}}^{L}, \tilde{X}_{l_{m}}^{L}\right]=-i(n-m) \tilde{X}_{l_{n+m}}^{L}-\frac{i}{12}\left(c n^{3}-c^{\prime} n\right) \Xi .
$$

Duality on (3.2) finally leads to the following quantization form:

$$
\begin{aligned}
\Theta= & \frac{-i}{24}\left(k^{3} c-k c^{\prime}\right) l_{-k} d l_{k}-\frac{c}{24} k^{2}\left(\sum_{n_{1}+n_{2}=-k}(-i)^{2} n_{1} n_{2} l_{n_{1}} l_{n_{2}}+\ldots\right. \\
& \left.+\sum_{n_{1}+\ldots+n_{j}=-k}(-i)^{j} n_{1} \ldots n_{j} l_{n_{1}} \ldots l_{n_{j}}\right) d l_{k}+\ldots+d \varphi .
\end{aligned}
$$

The characteristic subalgebra $\mathscr{G}_{\Theta}$ of $\Theta$ is the left subalgebra of $\widetilde{\mathscr{G}}$, a basis of which generates the characteristic module of $\Theta$, i.e., the vector fields on $\operatorname{diff} S^{1}$ such that:

$$
i_{\tilde{X}} d \Theta=0=i_{\tilde{X}} \Theta .
$$

In other words, $\mathscr{G}_{\Theta}$ is the kernel of the Lie algebra cocycle. The actual structure of $\mathscr{G}_{\theta}$ depends on the particular values of $c \neq 0$ and $c^{\prime}$. We find the following possibilities:

$$
\mathscr{G}_{\Theta}= \begin{cases}\left\langle\tilde{X}_{l_{0}}^{L}\right\rangle & \text { if } c^{\prime} / c \neq \mathbb{N}^{2}, \\ \left\langle\tilde{X}_{l_{0}}^{L}, \tilde{X}_{l_{-r}}^{L}, \tilde{X}_{l_{+r}}^{L}\right\rangle \approx s L^{(r)}(2, \mathbb{R}) & \text { if } \quad c^{\prime} / c=r^{2} \in \mathbb{N}^{2} .\end{cases}
$$

The corresponding symplectic manifolds ${ }^{1}\left(\operatorname{diff} S^{1}, d \Theta\right) / \mathscr{G}_{\Theta} \oplus u(1)$ will be

$$
\begin{gathered}
\left(\operatorname{diff} S^{1}, d \Theta\right) /\left\langle\tilde{X}_{l_{0}}^{L}\right\rangle, \\
\left(\operatorname{diff} S^{1}, d \Theta\right) / s L^{(r)}(2, \mathbb{R}), \quad r=1,2,3, \ldots
\end{gathered}
$$

respectively.

\footnotetext{
${ }^{1}$ The 2-form $d \Theta$ on $\operatorname{diff} S^{1} / S^{1}$ parametrized by $\left(c, c^{\prime}\right)$ turns out to be the Kähler metric [19]. We must remark at this point that no global complexification can be achieved on the Virasoro group [20]. The use of (local) complex coordinates (the group law is compatible with the rule $\left.l_{-n}^{*}=l_{n}\right)$ is only to simplify calculations and does not mean a lack of globality
} 
As in the general case the integral curves of the generators in $\mathscr{G}_{\Theta}$ can be interpreted as generalized equations of motion. In GAQ the Noether invariants are given by $i_{\tilde{X}^{R}} \Theta$ [5] which close on the same Poisson algebra as the respective right-invariant vector fields, i.e.,

$$
\left\{i_{\tilde{X}_{(a)}^{R}} \Theta, i_{\tilde{X}_{(b)}^{R}} \Theta\right\}=i_{\left[\tilde{X}_{(a)}^{R}, \tilde{X}_{(b)]}^{R}\right.} \Theta .
$$

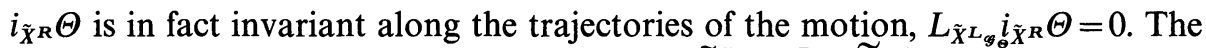
calculation of the right-invariant vector fields, $\widetilde{X}^{R} \in \mathscr{X}^{R}$ (diff $S^{1}$ ) is straightforward. By using (2.7) and (2.15) we get

$$
\begin{aligned}
\tilde{X}_{l_{k}}^{R}= & \frac{\partial}{\partial l_{k}}+i(m-k) l_{m-k} \frac{\partial}{\partial l_{m}} \\
& -\frac{c}{24} k^{2}\left\{(-i)(-k) l_{-k}+\frac{(-i)^{2}}{2} \sum_{n_{1}+n_{2}=-k} n_{1} n_{2} l_{n_{1}} l_{n_{2}}+\ldots\right. \\
& \left.+\frac{(-i)^{j}}{j} \sum_{n_{1}+\ldots+n_{j}=-k} n_{1} \ldots n_{j} l_{n_{1}} \ldots l_{n_{j}}+\ldots\right\} \Xi \\
& -\frac{c^{\prime}}{24} i(-k) l_{-k} \Xi .
\end{aligned}
$$

Equation (3.9) permits us in particular to obtain an explicit expression for the classical Hamiltonian $i_{\tilde{X}_{0_{0}}^{R}} \Theta$,

$$
\begin{aligned}
i_{\tilde{X}_{l_{0}}^{R}} \Theta & =\frac{1}{24} m\left(m^{3} c-m c^{\prime}\right)\left|l_{m}\right|^{2}+\frac{c}{24} m^{3}\left\{\sum_{n_{1}+n_{2}=-m}(-i)^{3} n_{1} n_{2} l_{n_{1}} l_{n_{2}} l_{m}+\ldots\right. \\
& \left.+\sum_{n_{1}+n_{2}+\ldots+n_{j}=-m}(-i)^{j+1} n_{1} \ldots n_{j} l_{n_{1}} \ldots l_{n_{J}} l_{m}+\ldots\right\},
\end{aligned}
$$

from which a study of positivity can be made along similar lines to those in ref. [2]. The critical points $p$ of $H=i_{\hat{X}_{R=0}^{R}} \Theta$ on the symplectic (solution) manifold, $\left.d H\right|_{P}=0$ satisfy

$$
\left.i_{\tilde{x}_{l o}^{R}} d \Theta\right|_{p}=0,
$$

and then

$$
\left.\tilde{X}_{l_{0}}^{R}\right|_{p}=0,
$$

for $d \Theta$ is not degenerate on the symplectic manifold. Therefore the critical points $p$ are left fixed under the left action of the $l_{0}$-subgroup. Looking now at (2.7) it is easy to see that the only critical points $p$ are those coming from the identity of the group when projected onto the symplectic manifolds. The stable critical points appear for diff $S^{1} /\left\langle l_{0}\right\rangle\left(c^{\prime} / c \neq \mathbb{N}^{2}\right)$ or $\operatorname{diff} S^{1} / S L^{(1)}(2, \mathbb{R})\left(c^{\prime} / c=1\right)$ and the Hamiltonian (3.10) is positive if $c>0$ and $c \geqq c^{\prime}$.

\section{Group Quantization and Virasoro Representations}

The GAQ idea is to perform the quantization from the Virasoro group itself rather than its symplectic manifolds (coadjoint orbits) (3.7). We do not need to start with 
any truly symplectic manifold. In this respect our point of view is different from that of the standard Geometric Quantization approach applied to the coadjoint orbits [2]. Nevertheless both formulations have many common points.

In order to quantize the group diff $S^{1}$ we start with the complex functions on it $\mathscr{F}\left(\right.$ diff $\left.S^{1}, \mathbb{C}\right)$. A naive quantization (prequantization or Bohr quantization) is achieved from the right-invariant vector fields acting on $\mathscr{F}\left(\operatorname{diff} S^{1}, \mathbb{C}\right)$. This representation is highly reducible, and it is for that reason that a proper quantization mechanism must be followed. One can in fact check that there are non-trivial operators, $\tilde{X}^{L}$ indeed, which commute with the representation $\tilde{X}^{R}\left(\left[\tilde{X}^{R}, \tilde{X}^{L}\right]=0\right.$ is well known to be a general group feature). Then reducing the representation means seeking a maximal set of compatible conditions on $\mathscr{F}\left(\operatorname{diff} S^{1}, \mathbb{C}\right)$ trivializing the effect of $\mathscr{X}^{L}\left(\operatorname{diff} S^{1}, \mathbb{C}\right)$. For instance the operator $\tilde{X}_{(\zeta)}^{R} \equiv \Xi$ can be made trivial by imposing the so-called $U(1)$-equivariance condition already found in the traditional Geometric Quantization formalism [1]. That is to say, $\Xi \Psi=i \Psi$. The next step is to impose the maximum number of compatible conditions of the form $\tilde{X}^{L} \Psi=0$. This is actually accomplished by means of a polarization.

Unlike the customary definition of polarization [1] where a certain unwanted ambiguity is present [14], in our quantization formalism the definition of polarization is constrained by the structure of $\mathscr{G}_{\Theta}$. A Full Polarization $\mathscr{F} \mathscr{P}$ (respectively Polarization $\mathscr{P}$ ) is a maximal left-subalgebra containing (respectively a proper subalgebra of) $\mathscr{G}_{\circledast}[11,12]$ and excluding $\Xi$. This definition generalizes that of Geometric Quantization. In some special cases an obstruction to the Full Polarization can occur. This obstruction is associated with the existence of gauge transformations in anomalous theories relating two different polarizations [19]. From the classification (3.6) we find the following non-equivalent Full Polarizations and Polarizations.

For $\mathscr{G}_{\Theta}=\left\langle\tilde{X}_{l_{0}}^{L}\right\rangle$ the Full Polarization is

$$
\mathscr{F} \mathscr{P}=\mathscr{P}=\left\langle\tilde{X}_{l_{n} \leqq 0}^{L}\right\rangle .
$$

For $\mathscr{G}_{\Theta}=S L^{(1)}(2, \mathbb{R})$ the Full Polarization is

$$
\mathscr{F} \mathscr{P}^{(1)}=\mathscr{P}^{(1)}=\left\langle\tilde{X}_{l_{n} \leqq 1}^{L}\right\rangle .
$$

In both (4.1) and (4.2) cases Full Polarizations and Polarizations coincide. For $\mathscr{G}_{\Theta}=S L^{(r)}(2, \mathbb{R})_{r \geqq 2}$ the Full Polarization is

$$
\mathscr{F} \mathscr{P}^{(r)}=\left\langle\tilde{X}_{l_{k r}}^{L}\right\rangle, \quad k=-1,0,1,2,3, \ldots,
$$

but the Polarization is different ${ }^{2}$ :

$$
\mathscr{P}^{(r)}=\left\langle\tilde{X}_{l_{n} \leqq 0}^{L}\right\rangle .
$$

From now on we only deal with the two different Polarizations $\mathscr{P}^{(r \geqq 2)}=\left\langle\tilde{X}_{l_{n} \leqq 0}^{L}\right\rangle$ and $\mathscr{P}^{(1)}=\left\langle\tilde{X}_{l_{n} \leqq 1}^{L}\right\rangle$ which are complete in the sense that they have "half" the Virasoro generators. We neglect the Full Polarization $\mathscr{F}_{\mathscr{P}^{(r \geqq 2)}}$ because we think that it would lead to a set of non-standard highly reducible representations. The use of $\mathscr{P}^{(r \geqq 2)}$ will lead, on the contrary, to the highest-weight Virasoro

${ }^{2}$ In a different language these results can be expressed by saying that the only symplectic orbits of (3-7) which are Kähler manifolds are diff $S^{1} / S^{1}$ and diff $S^{1} / S L^{1}(2, \mathbb{R})[2]$ 
representations with $c^{\prime} / c=r^{2}, r=2,3, \ldots$ In terms of $c$ and $h=\left(c-c^{\prime}\right) / 24$ the relation $c^{\prime} / c=r^{2}$ is $h=-c\left(r^{2}-1\right) / 24$ and is known to correspond to non-unitary representations.

We define the wave functions as the $U(1)$-equivariant functions on $\operatorname{diff} S^{1}$ $(\Xi \Psi=i \Psi)$ satisfying the polarization conditions

$$
\begin{array}{lll}
\tilde{X}_{l_{n} \leqq 0}^{L} \Psi=0 & \text { if } & c \neq c^{\prime}, \\
\tilde{X}_{l_{n} \leqq 1}^{L} \Psi=0 & \text { if } & c=c^{\prime} .
\end{array}
$$

We restrict ourselves to the case $c \neq c^{\prime}$ because that of $c=c^{\prime}$ (the difference being the extra polarization condition $\tilde{X}_{l_{+1}}^{L} \Psi=0$ ), can formally be obtained from the former by putting $c=c^{\prime}$ everywhere at the end of the calculus.

Equations (4.4a) can be exactly solved to any order. The polarized wave functions factorize into a product of basic functions $W$ and $\left\{l_{n}\right\}, n=1,2,3, \ldots$, one of which, the weight function $W$, is a common factor in all states. We find

$$
\begin{aligned}
|0\rangle & =\zeta W \quad \text { (the vacuum) } \\
\left|l_{n}\right\rangle & =\zeta W\left\{l_{n}\right\} \\
\ldots & \cdots \\
\ldots & \ldots \\
\left|l_{n_{1}} \ldots l_{n_{j}}\right\rangle & =\zeta W\left\{l_{n_{1}}\right\}\left\{l_{n_{2}}\right\} \ldots\left\{l_{n_{j}}\right\},
\end{aligned}
$$

where the explicit expressions for $W$ and $\left\{l_{n}\right\}$ are given below. Here we just write the terms which are relevant in computing the Lie algebra operators. The remaining part of each function can be systematically computed from the exact expression of the left-invariant vector fields (3.2). The superposition property of the states can be proved by exactly the same procedure used in ref. [11]. Up to the factor $\zeta$ the vacuum is given by

$$
\begin{aligned}
W= & +\frac{1}{24}\left\{\sum_{\substack{n_{1}>0 \\
q_{1}=n_{1}}} Q^{(1)}\left(n_{1}\right) l_{n_{1}} l_{-q_{1}}+\sum_{\substack{n_{1}, n_{2}>0 \\
q_{2}=n_{1}+n_{2}}} Q^{(2)}\left(n_{1}, n_{2}\right) l_{n_{1}} l_{n_{2}} l_{-q_{2}}+\ldots\right. \\
& \left.+\sum_{\substack{n_{1}, \ldots, n_{j}>0 \\
q_{j}=n_{1}+\ldots+n_{j}}} Q^{(j)}\left(n_{1}, \ldots, n_{j}\right) l_{n_{1}} \ldots l_{n_{j}-q_{j}} l_{-\ldots}+\ldots\right\} \\
Q^{(1)}\left(n_{1}\right)= & -i c q_{1}^{2}\left(-i n_{1}\right)+i c^{\prime}\left(-i n_{1}\right), \\
Q^{(2)}\left(n_{1}, n_{2}\right)= & -\left(-i q_{2}\right) Q^{(1)}\left(n_{1}\right)-i c \frac{q_{2}^{2}}{2 !}(-i)^{2} P^{(2)}\left(n_{1}, n_{2}\right)+i c^{\prime} \frac{\left(-i q_{2}\right)^{2}}{2 !} \\
Q^{(3)}\left(n_{1}, n_{2}, n_{3}\right)= & -\left(-i q_{3}\right) Q^{(2)}\left(n_{1}, n_{2}\right)-\frac{\left(-i q_{3}\right)^{2}}{2 !} Q^{(1)}\left(n_{1}\right) \\
& -i c \frac{q_{3}^{2}}{3 !}(-i)^{3} P^{(3)}\left(n_{1}, n_{2}, n_{3}\right)+i c^{\prime} \frac{\left(-i q_{3}\right)^{3}}{3 !} \\
\ldots & \\
\ldots & -\sum_{k=1}^{j-1} \frac{\left(-i q_{j}\right)^{k}}{k !} Q^{(j-k)}\left(n_{1}, \ldots, n_{j-k}\right)+(-i)^{j+1} \\
\times & {\left[\frac{c q_{j}^{2}}{j !} P^{(j)}\left(n_{1}, \ldots, n_{j}\right)-c^{\prime} \frac{q_{j}^{j}}{j !}\right] }
\end{aligned}
$$


where the polynomials $P$ where defined by (2.10), (2.11). For the functions $\left\{l_{q}\right\}$ we write

$$
\begin{aligned}
\left\{l_{q}\right\}= & l_{q}+\sum_{\substack{n_{1}>0 \\
n_{1}>q}} R^{(1)}\left(n_{1} ; q\right) l_{n_{1}} l_{q-n_{1}} \\
& +\sum_{\substack{n_{1}, n_{2}>0 \\
n_{1}+n_{2}>q}} R^{(2)}\left(n_{1}, n_{2} ; q\right) l_{n_{1}} l_{n_{2}} l_{q-n_{1}-n_{2}}+\ldots \\
& +\sum_{\substack{n_{1}, \ldots, n_{j}>0 \\
n_{1}+n_{2}+\ldots+n_{j}>q}} R^{(j)}\left(n_{1}, \ldots, n_{j} ; q\right) l_{n_{1}} \ldots l_{n_{j}} l_{q-n_{1}-\ldots-n_{j}}+\ldots, \\
R^{(1)}\left(n_{1} ; q\right)= & i\left(n_{1}-q\right) ; \\
R^{(2)}\left(n_{1}, n_{2} ; q\right)= & i\left(n_{1}+n_{2}-q\right)\left(n_{1}-q\right)-\frac{(-i)^{2}}{2 !}\left(n_{1}+n_{2}-q\right), \\
R^{(3)}\left(n_{1}, n_{2}, n_{3} ; q\right)= & i\left(n_{1}+n_{2}+n_{3}-q\right) R^{(2)}\left(n_{1}, n_{2} ; q\right) \\
& -\frac{(-i)^{2}}{2 !}\left(n_{1}+n_{2}+n_{3}-q\right)^{2}-\frac{(-i)^{3}}{3 !}\left(n_{1}+n_{2}+n_{3}-q\right)^{3} \\
\ldots & \\
R^{(j)}\left(n_{1}, \ldots, n_{j} ; q\right)= & \sum_{s=1}^{j} \frac{(-i)^{s}}{s !}\left(n_{1}+\ldots+n_{j}-q\right)^{s} R^{(j-s)}\left(n_{1}, \ldots, n_{j-s} ; q\right) .
\end{aligned}
$$

The group representations (parametrized by $c$ and $c^{\prime}$ ) could now be explicitly obtained from the polarized functions and the group law by means of the finite lefttranslations

$$
L_{\tilde{g}^{\prime}} \Psi(\tilde{g})=\Psi\left(\tilde{g}^{\prime} * \tilde{g}\right), \quad \forall \tilde{g}^{\prime}, \tilde{g} \in \operatorname{diff} S^{1} .
$$

We only make explicit the Lie algebra action through the right-invariant vector fields. From (3.9), (4.6-7) we obtain exactly

$$
\begin{aligned}
& \tilde{X}_{l_{-k}}^{R}|0\rangle=\frac{1}{24}\left\{\sum_{\substack{k=1 \\
j}} \sum_{\substack{n_{1}, \ldots, n_{j}>0 \\
n_{1}+\ldots+n_{j}=k}} Q^{(j)}\left(n_{1}, \ldots, n_{j}\right)\left|l_{n_{1}} \ldots l_{n_{j}}\right\rangle\right. \\
& +i \sum_{s=0}^{k-1}(k-s) \sum_{j=1}^{s} \sum_{\substack{n_{1}, \ldots, n_{j}>0 \\
n_{1}+\ldots+n_{j}=s}} Q^{(j)}\left(n_{1}, \ldots, n_{j}\right)\left|l_{k-s} l_{n_{1}} \ldots l_{n_{j}}\right\rangle \\
& \left.+c k^{2} \sum_{j=1}^{k} \sum_{\substack{n_{1}, \ldots, n_{j}>0 \\
n_{1}+\ldots+n_{j}=k}} \frac{(-i)^{j+1}}{j} n_{1} \ldots n_{j}\left|l_{n_{1}} \ldots l_{n_{j}}\right\rangle+c^{\prime} k\left|l_{k}\right\rangle\right\} \text {, } \\
& \tilde{X}_{l_{-k}<0}^{R}\left|l_{q}\right\rangle=\sum_{j=1}^{k+q} \sum_{\substack{n_{1}, \ldots, n_{j}>0 \\
n_{1}+\ldots+n_{j}=k+q}} R^{(j)}\left(n_{1}, \ldots, n_{j} ; q\right) \\
& \times\left|l_{n_{1}} \ldots l_{n_{j}}\right\rangle+i(k+q)\left|l_{q+k}\right\rangle \\
& +i \sum_{s=0}^{k-1}(k-s) \sum_{j=1}^{s+q} \sum_{\begin{array}{c}
n_{1}, \ldots, n_{j}>0 \\
n_{1}+\ldots+n_{j}=s+q
\end{array}} R^{(j)}\left(n_{1}, \ldots, n_{j}\right) \\
& \left.\left.\times\left|l_{k-s} l_{n_{1}} \ldots l_{n_{j}}\right\rangle+\left|l_{q} \tilde{X}_{l_{-k}}^{R}\right| 0\right\rangle\right\rangle, \\
& \tilde{X}_{l_{k \geqq 0}}^{R}|0\rangle=0, \\
& \tilde{X}_{l_{k \geqq 0}}^{R}\left|l_{q}\right\rangle=\delta_{k, q}|0\rangle+i(q-k)\left|l_{q-k}\right\rangle .
\end{aligned}
$$


The action of the basic operators $\tilde{X}_{l_{k}}^{R}$ on any other state $\left|l_{n_{1}} \ldots l_{n_{j}}\right\rangle$ follows from $(4.9 \mathrm{a}-\mathrm{c})$ because of the factorization property of the polarized functions. The commutation relations among the right-invariant vector fields

$$
\left[\tilde{X}_{l_{n}}^{R}, \tilde{X}_{l_{m}}^{R}\right]=i(n-m) \tilde{X}_{l_{n+m}}^{R}+\frac{i}{12}\left(c n^{3}-c^{\prime} n\right) \delta_{n,-m} \Xi
$$

are converted to the usual Virasoro commutators, $\left[\hat{L}_{n}, \hat{L}_{m}\right]=(n-m) \hat{L}_{n+m}$ $+c\left(n^{3}-n\right) / 12$, with the redefinition

$$
\hat{L}_{n \neq 0} \equiv-i \tilde{X}_{l_{n}}^{R}, \quad \hat{L}_{0}=-i\left(\tilde{X}_{l_{0}}^{R}+h \Xi\right),
$$

where $h=\left(c-c^{\prime}\right) / 24$ is the vacuum expectation value of $\hat{L}_{0}$.

The above expressions (4.9-11) can be seen to constitute a Virasoro representation on the space of (polarized) wave functions. Had we taken the polarization $\mathscr{P}^{(1)}\left(c=c^{\prime} \Leftrightarrow h=0\right)$ we would have obtained a set of states of the form $\left|l_{n_{1}} \ldots l_{n_{j}}\right\rangle$, $n_{k} \geqq 2$. In both cases the polarized functions are linearly independent, and the associated partition functions are

$$
\begin{gathered}
\operatorname{Tr} q^{\hat{L}_{0}}=q^{n} \prod_{n=1}^{\infty} \frac{1}{1-q^{n}} \quad \text { if } \quad h \neq 0, \\
\operatorname{Tr} q^{\hat{L}_{0}}=\prod_{n=2}^{\infty} \frac{1}{1-q^{n}} \quad \text { if } \quad h=0,
\end{gathered}
$$

which coincide with those obtained in the first reference in ref. [2], for the "quantizable" orbits diff $S^{1} / S^{1}$ and $\operatorname{diff} S^{1} / S L^{(1)}(2, \mathbb{R})$, by using the fixed point theorem. At this moment, a very careful analysis of the representations (4.9) in relation with the standard theory for the Virasoro group and, in particular, with the GQCO method, is required.

One of the main properties of the representations here obtained is the absence of null vectors, i.e., polarized functions on the group vanishing under all the annihilation operators. This property is stated by the following

Proposition. The only (polarized) wave function on the group satisfying $\widetilde{X}_{l_{n} \geqq 0}^{R} \Psi=0$ is the weight function $\zeta W$.

The Proof immediately follows from the fact that we know the operators $\tilde{X}_{l_{n}}^{R}$ explicitly. In fact, the equations $\tilde{X}_{l_{n} \geqq 0}^{R} \Psi=0$ can be integrated explicitly with the general solution

$$
\Psi=C_{0} \zeta \tilde{W}+C_{n_{1}}\left[l_{n_{1}<0}\right]+C_{n_{1} n_{2}}\left[l_{n_{1}<0} l_{n_{2}<0}\right]+\ldots
$$

where

$$
\begin{aligned}
{\left[l_{n_{1}}\right] } & =\zeta \tilde{W}\left\{l_{n_{1}}+O(2)\right\}, \\
{\left[l_{n_{1}} l_{n_{2}}\right] } & =\zeta \tilde{W}\left\{l_{n_{1}}+O(2)\right\}\left\{l_{n_{2}}+O(2)\right\} \\
\dddot{W} & =1+O(2) .
\end{aligned}
$$


It is clear from the general solution (4.13) that the only solution which can be in turn a polarized function is $\zeta \tilde{W}$ since the leading terms of the functions $\left[l_{n_{1}} \ldots l_{n_{k}}\right]$, unlike the functions (4.5), have negative indices. However, the analytic function $\zeta \tilde{W}$ satisfies exactly the same linear differential system $\left(\widetilde{X}_{l_{n \geq 0}}^{R} \zeta \widetilde{W}=0\right)$ as $\zeta W$ [see (4.9c)] and coincide with it. This result can also be tested easily by direct calculation.

A crucial point to be remarked is that, unlike for finite-dimensional compact semisimple groups, the space of polarized functions on the group or, equivalently, the space of holomorphic sections of the corresponding line bundle in the Kähler Geometric Quantization scheme, is not in general irreducible (in this respect the Virasoro group is different from the loop groups on compact Lie groups for which the Borel-Weil-Bott theorem [20] still applies). Indeed, the invariant subspace

$$
\left.\mathscr{H}_{(c, h)} \equiv\left\langle\tilde{X}_{l_{n_{j}}}^{R} \ldots \tilde{X}_{l_{n_{1}}}^{R} \mid \zeta W\right\rangle\right\rangle, \quad n_{k}>0, j=1,2,3, \ldots
$$

is a proper subspace for certain values of $c$ and $h$ (see later). A corollary of the above proposition is that (4.15) is irreducible. Therefore, the action of the right-invariant vector fields on $\mathscr{H}_{(c, h)}$ provides a highest weight representation without null vectors. As a consequence of the irreducibility of $\mathscr{H}_{(c, h)}$ (no null vector is present), the possible linear relations among states in (4.15) appear automatically and no additional quotient has to be taken. In other words, the actual values of $c$ and $h$ for which $\mathscr{H}_{(c, h)}$ is a proper subspace of the set of polarized functions will emerge in a straightforward way and coincide with those annihilating the Kac determinant.

We want to insist on the difference between our construction of (4.15) and the analogous construction (Verma module) in the standard abstract theory. There one starts with an abstract vector state $|\Omega\rangle$ with lowest $\hat{L}_{0}$-eigenvalue $h$ and satisfying $\hat{L}_{m>0}|\Omega\rangle=0$, and then constructs a formal (Verma) module by linear combination of

$$
\prod_{n=1}^{\infty} \hat{L}_{-n}^{c_{n}}|\Omega\rangle
$$

The formal states (4.14) themselves may firstly be considered as generators of a linear space and then constrained according to the special values of $c$ and $h$. It is well known that the Virasoro Verma moduli for $c \leqq 1$ posess null vectors [16] (more precisely, for $c>1$ the Verma modules are irreducible, for $c=1$ there is a null vector on level $n$ given by $h=(n-1)^{2} / 4$, and for $c<1$ there are more than one) and the irreducible representations are quotients of Verma moduli by the null vectors.

On the contrary, our construction does not start with abstract states but rather with explicit functions on which the differential operators $\tilde{X}^{R}$ do act. Thus, the possible linear relations among states in (4.15) will appear automatically. Let us illustrate this fact with a few examples, and we will see how the values at which the linear relations happen are those for which null vector states are found in the standard representation theory [16].

To this end we first write the creation operators to the lowest orders: 


$$
\begin{aligned}
\tilde{X}_{l-k<0}^{R}|0\rangle= & \frac{1}{24}\left\{\left(-c k^{3}+c^{\prime} k\right)\left|l_{k}\right\rangle+i \sum_{\substack{n_{1}, n_{2}>0 \\
n_{1}+n_{2}=k}}\left[-k\left(-c n_{1}^{3}+c^{\prime} n_{1}\right)\right.\right. \\
& \left.+\frac{k^{2}}{2 !}\left(n_{1}^{2}+n_{2}^{2}+2 n_{1} n_{2}\right) c-\frac{1}{2 !}\left(n_{1}+n_{2}\right)^{2} c^{\prime}\right]\left|l_{n_{1}} l_{n_{2}}\right\rangle \\
& +\sum_{\substack{n_{1}, n_{2}, n_{3}>0 \\
n_{1}+n_{2}+n_{3}=k}}\left[-k\left[\left(n_{1}+n_{2}\right)\left(-c n_{1}^{3}+c^{\prime} n_{1}\right)\right.\right. \\
& \left.+\frac{1}{2 !}\left(n_{1}+n_{2}\right)^{2}\left(n_{1}^{2}+n_{2}^{2}+n_{1} n_{2}\right) c-\frac{1}{2 !}\left(n_{1}+n_{2}\right)^{2} c^{\prime}\right] \\
& \frac{k^{2}}{2 !}\left(-c n_{1}^{3}+c^{\prime} n_{1}\right)+\frac{k^{2}}{2 !} P^{(3)}\left(n_{1}, n_{2}, n_{3}\right) c-\frac{k^{3}}{3 !} c^{\prime}\left[\left|l_{n_{1}} l_{n_{2}} l_{n_{3}}\right\rangle\right. \\
& +i \sum_{s=0}^{k-1}(k-s)\left[\left(-c s^{3}+c^{\prime} s\right)\left|l_{k-s} l_{s}\right\rangle\right. \\
& \left.+i \sum_{n_{1}+n_{2}=s}\left[s\left(-c n_{1}^{3}+c^{\prime} n_{1}\right)+\frac{s^{2}}{2 !}\left(n_{1}^{2}+n_{2}^{2}+n_{1} n_{2}\right) c-\frac{s^{2}}{2 !} c^{\prime}\right]\left|l_{k-s} l_{n_{1}} l_{n_{2}}\right\rangle+\ldots\right] \\
& +c k^{2}\left[-k\left|l_{k}\right\rangle+\frac{i}{2} n_{1} n_{2}\left|l_{n_{1}} l_{n_{2}}\right\rangle\right. \\
& \left.\left.+\frac{1}{3} n_{1} n_{2} n_{3}\left|l_{n_{1}} l_{n_{2}} l_{n_{3}}\right\rangle+\ldots\right]+c^{\prime} k\left|l_{k}\right\rangle\right\}, \\
&
\end{aligned}
$$$$
\tilde{X}_{l-k<0}^{R}\left|l_{q}\right\rangle=i k\left|l_{q+k}\right\rangle-\sum_{\substack{n_{1}+n_{2}=q+k \\ n_{1}>q}}\left[k\left(n_{1}-q\right)-\frac{k^{2}}{2 !}\right]\left|l_{n_{1}} l_{n_{2}}\right\rangle
$$$$
-\sum_{\substack{n_{1}+n_{2}+n_{3}=q+k \\ n_{1}, n_{2}>q}}\left[( - i ) k \left(i\left(n_{1}+n_{2}-q\right) i\left(n_{1}-q\right)\right.\right.
$$$$
\left.\left.+\frac{1}{2 !}\left(n_{1}+n_{2}-q\right)^{2}\right)-\frac{1}{2 !} k^{2} i\left(n_{1}-q\right)+i \frac{k^{3}}{3 !}\right]
$$$$
\times\left|l_{n_{1}} l_{n_{2}} l_{n_{3}}\right\rangle+\ldots+i(k+q)\left|l_{q+k}\right\rangle
$$$$
+i \sum_{s=0}^{k-1}(k-s)\left[i s\left|l_{k-s} l_{q+s}\right\rangle+\sum_{n_{1}+n_{2}=q+s}\left(-s\left(n_{1}-q\right)+\frac{s^{2}}{2}\right)\right.
$$$$
\left.\left.\left.\times\left|l_{k-s} l_{n_{1}} l_{n_{2}}\right\rangle+\ldots\right]+\left|l_{q} \tilde{X}_{l-k}^{R}\right| 0\right\rangle\right\rangle \text {. }
$$

The vector state defined as $\tilde{X}_{l^{-1}}^{R}|0\rangle$ is the following wave function,

$$
\tilde{X}_{l-1}^{R}|0\rangle=\frac{1}{12}\left(-c+c^{\prime}\right)\left|l_{1}\right\rangle,
$$

which is automatically zero if $c=c^{\prime}$ (or $h=0$ ).

The next examples are less trivial. The irreducible space of level two is generated by the functions $\tilde{X}_{l_{-2}}^{R}|0\rangle$ and $\left(\tilde{X}_{l_{-1}}^{R}\right)^{2}|0\rangle$, and the question arises as to 
whether or not these wave functions are proportional. With the help of expressions (4.18) one can compute both wave functions:

$$
\begin{gathered}
\tilde{X}_{l_{-2}}^{R}|0\rangle=-\frac{1}{12}\left(8 c-2 c^{\prime}\right)\left|l_{2}\right\rangle-\frac{i}{24}\left(-5 c-c^{\prime}\right)\left|l_{1} l_{1}\right\rangle, \\
\tilde{X}_{l_{-1}}^{R} \tilde{X}_{l_{-1}}^{R}|0\rangle=-\frac{c-c^{\prime}}{12}\left[3 i\left|l_{2}\right\rangle+\frac{1}{2}\left|l_{1} l_{1}\right\rangle-\frac{1}{12}\left(c-c^{\prime}\right)\left|l_{1} l_{1}\right\rangle\right] .
\end{gathered}
$$

They are proportional, i.e., $\left(\tilde{X}_{l_{-2}}^{R}+\lambda\left(\tilde{X}_{l_{-1}}^{R}\right)^{2}\right)|0\rangle=0$, if

$$
h=\frac{1}{16}[5-c \pm \sqrt{(c-1)(c-25)}] .
$$

Finally we consider the level three. The irreducible space of level three is generated by the functions $\widetilde{X}_{l_{-3}}^{R}|0\rangle, \widetilde{X}_{l_{-2}}^{R} \tilde{X}_{l_{-1}}^{R}|0\rangle$, and $\left(\widetilde{X}_{l_{-1}}^{R}\right)^{3}|0\rangle$ :

$$
\begin{aligned}
\tilde{X}_{l_{-3}}^{R}|0\rangle= & \frac{1}{24}\left\{\left(-54 c+6 c^{\prime}\right)\left|l_{3}\right\rangle+i\left(44 c+4 c^{\prime}\right)\left|l_{1} l_{2}\right\rangle+8 c\left|l_{1} l_{1} l_{1}\right\rangle,\right. \\
\tilde{X}_{l_{-2}}^{R} \tilde{X}_{l^{-1}}^{R}|0\rangle= & \frac{1}{12}\left(-c+c^{\prime}\right)\left[5 i\left|l_{3}\right\rangle+\left(1-\frac{1}{12}\left(8 c-2 c^{\prime}\right)\right)\left|l_{1} l_{2}\right\rangle\right. \\
& \left.+i\left(\frac{1}{6}+\frac{1}{24}\left(5 c+c^{\prime}\right)\right)\left|l_{1} l_{1} l_{1}\right\rangle\right], \\
\left(\tilde{X}_{l_{-1}}^{R}\right)^{3}|0\rangle= & \frac{1}{12}\left(-c+c^{\prime}\right)\left[-12\left|l_{3}\right\rangle+\left(6 i+\frac{i 3}{4}\left(-c+c^{\prime}\right)\right)\left|l_{1} l_{2}\right\rangle\right. \\
& \left.+\left(1+\frac{1}{8}\left(-c+c^{\prime}\right)+\frac{1}{(12)^{2}}\left(-c+c^{\prime}\right)^{2}\right)\left|l_{1} l_{1} l_{1}\right\rangle\right] .
\end{aligned}
$$

They are proportional, i.e., $\left(\widetilde{X}_{l_{-3}}^{R}+\mu \widetilde{X}_{l_{-2}}^{R} \tilde{X}_{l_{-1}}^{R}+\mu^{\prime}\left(\widetilde{X}_{l_{-1}}^{R}\right)^{3}\right)|0\rangle=0$ for

$$
h=\frac{1}{6}[7-c \pm \sqrt{(c-1)(c-25)}]
$$

or, the values $[(4.20)]$ which are the induced ones from the previous level and correspond to relations of the form $\tilde{X}_{l_{-1}}^{R}\left(\tilde{X}_{l_{-2}}^{R}+\lambda\left(\tilde{X}_{l_{-1}}^{R}\right)^{2}\right)|0\rangle=0$.

\section{Final Comments and Comparison with the Coadjoint Orbits Method}

Let us summarize in a few lines. Starting from the Lie algebra of the Virasoro group we have constructed a local group law to which we have applied our GAQ. This method provides a functional space of polarized functions on the Virasoro group constituting a Virasoro Lie algebra module or, even more, a carrier space for the finite action of the group. As a non-trivial result we find that unlike the finitedimensional compact semisimple Lie group this space of polarized functions is not irreducible. However, the reduction is achieved in a straightforward manner by just taking the invariant subspace $\mathscr{H}_{(c, h)}$ which is irreducible irrespective of the concrete values of $c$ and $h$. Discrete values of $c$ and $h$, precisely those for which the Kac determinant is zero, characterize the representations whose carrier space $\mathscr{H}_{(c, h)}$ is a proper subspace of the space of polarized functions. Nevertheless we are always rid of null vector states. The fact that $\mathscr{H}_{(c, h)}$ does not coincide, in general, with the space of polarized functions finds its origin in the non-compactness of the 

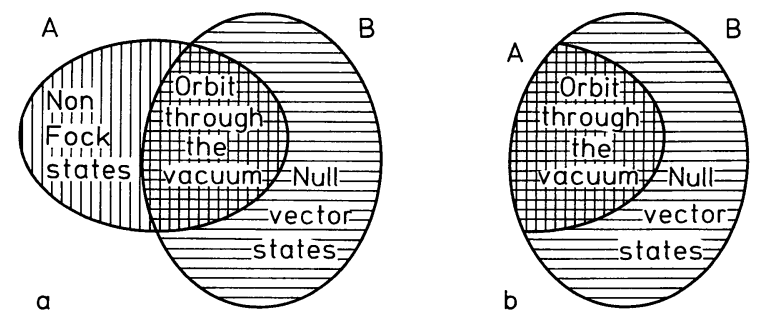

Fig. 1. $\mathbf{A}$ is the space of polarized functions on the group, $\mathbf{B}$ the Verma module. $\mathbf{A} \cap \mathbf{B}$ is the irreducible carrier space for the group representations. In a) not all the polarized functions are obtained from the vacuum. In b) the whole space $\mathbf{A}$ is irreducible. a) Virasoro group. b) Compact groups (finite-dimensional semisimple groups and Loop groups)

Virasoro group. In the compact cases (finite-dimensional simple Lie groups and Loop groups [20], going from the local solutions of the polarization equations to the global ones restricts the space and make it irreducible (see Fig. 1).

One of the improvements of the method here developed with respect to the GQCO is the capability of providing the irreducible representations of the group for any value of $c$ and $h$. For the latter only the (Kähler) orbits diff $S^{1} / S^{1}$ and $\operatorname{diff} S^{1} / S L^{(1)}(2, \mathbb{R})$ are quantizable (polarizable) and the partition function for these, coinciding with ours (4.12), corresponds to irreducible representations only for $c>1$. According to the strict rules of the Geometric Quantization one must conclude that this method is unable to get all the irreducible unitary representations (in particular the discrete series $0<c \leqq 1$ ) [21], as stated in the first reference of ref. [2].

In the light of the results obtained with the GAQ we have the strong feeling that what happens in the GQCO method for $c \leqq 1$ is simply that the representations obtained by means of the holomorphic sections are not irreducible and the full reduction could be accomplished by just considering the "orbit" through the vacuum of the enveloping algebra, rather than looking for a non-standard quantization of the non-polarizable co-adjoint orbits. ${ }^{3}$

Another open question concerns the scalar product. We also believe that the breakdown of square-integrability might be at $c=0$, where the unitarity ceases, instead of $c=1$, where the space of polarized functions (respectively holomorphic sections) stops being irreducible. This is something to be discussed more accurately once a proper scalar product will be defined. In principle, it seems natural to prolong the definition of the scalar product on the $S L(2, \mathbb{R})$ group (A.14) to the Virasoro group by means of the formal integral

$$
\left\langle\psi^{\prime}, \psi\right\rangle \equiv \int_{\text {diff } S^{1}}\left(\theta^{L^{\left(l_{0}\right)}} \wedge \theta^{L_{\left(l_{1}\right)}} \wedge \theta^{L_{(l-1)}} \wedge \theta^{L_{(12)}} \wedge \ldots\right) \psi^{\prime \dagger} \psi,
$$

where $\vartheta^{L()}$ are components of the left-invariant canonical 1-form $\vartheta^{L}$. The practical capability of this expression is under study.

We want to point out, as a final comment, that the mechanism which has led to the full reduction of the polarized functions on the Virasoro group is of general applicability and has already been used to obtain the irreducible representations of a supergroup, the BRST-supergroup, containing among its generators the $Q$-charge, the ghost and anti-ghost operators and the Virasoro constraints of

${ }^{3}$ Examples of non-polarizable, finite-dimensional symplectic manifolds have been given in reference [22] 
string theory. The critical dimension arises in the same way as the critical values $c$ and $h$ here [23].

\section{Appendix}

This appendix is devoted to a brief illustration of the Group Approach to Quantization formalism by means of a much simpler example: the $S L(2, \mathbb{R})$ subgroup (see ref. [14] where the dynamics on $S L(2, \mathbb{R})$ is interpreted either as that of a relativistic harmonic oscillator or as a particle moving on a $1+1$ anti de Sitter universe.

The group law we give for $S L(2, \mathbb{R})$ is written in a global fibre bundle chart of the fibration $S L(2, \mathbb{R}) \rightarrow S L(2, \mathbb{R}) / U(1)$. In this chart $\eta$ parametrizes the fibre $U(1)$ and $2 z, 2 z^{*}$ the hyperboloid $\Omega$. The law is

$$
\begin{aligned}
z^{\prime \prime} & =z^{\prime} \eta^{-2}+\kappa^{\prime} z+\frac{2 z}{1+\kappa}\left[z^{*} z^{\prime} \eta^{-2}+z^{*} z \eta^{2}\right], \\
z^{* \prime \prime} & =z^{* \prime} \eta^{2}+\kappa^{\prime} z^{*}+\frac{2 z^{*}}{1+\kappa}\left[z z^{*} \eta^{2}+z^{\prime} z^{*} \eta^{-2}\right], \\
\eta^{\prime \prime} & =\sqrt{\frac{2}{1+\kappa^{\prime \prime}}}\left\{\sqrt{\frac{1+\kappa}{2}} \sqrt{\frac{1+\kappa^{\prime}}{2}} \eta^{\prime} \eta-\sqrt{\frac{2}{1+\kappa}} \sqrt{\frac{2}{1+\kappa^{\prime}}} z^{*} z^{\prime} \eta^{*} \eta^{\prime}\right\}
\end{aligned}
$$

where $\kappa=\sqrt{1+4 z z^{*}}$ and $\kappa^{\prime \prime}=\kappa^{\prime} \kappa-2\left(z^{*} z^{\prime} \eta^{-2}+z^{* \prime} z \eta^{2}\right)$.

The fibration $S L(2, \mathbb{R}) \rightarrow S L(2, \mathbb{R}) / U(1)$ canonically defines a $\mathbb{Z}$-parametrized family of pseudo-extensions which are realized by means of coboundaries generated by linear functions on the $\eta$ parameter of the $U(1)$ structure subgroup. The group law for the $U(1)$ structure subgroup of the pseudo-extended group is $(\zeta=\exp i \varphi \in U(1))$ :

$$
\zeta^{\prime \prime}=\zeta^{\prime} \zeta\left(\eta^{\prime \prime} \eta^{-1} \eta^{-1}\right)^{N}, \quad N \in \mathbb{Z} .
$$

From (A.1) and (A.2) the left- and right-invariant vector fields are respectively

$$
\begin{gathered}
\tilde{X}_{(z)}^{L}=\kappa \frac{\partial}{\partial z}+\frac{2 i}{1+\kappa} z^{*} \frac{\partial}{\partial \theta}-i N \frac{z^{*}}{1+\kappa} \Xi \\
\tilde{X}_{\left(z^{*}\right)}^{L}=\kappa \frac{\partial}{\partial z^{*}}-\frac{2 i}{1+\kappa} z \frac{\partial}{\partial \theta}+i N \frac{z}{1+\kappa} \Xi, \\
\tilde{X}_{(\theta)}^{L}=\frac{\partial}{\partial \theta}-i z \frac{\partial}{\partial z}+i z^{*} \frac{\partial}{\partial z^{*}}, \\
\tilde{X}_{(\zeta)}^{L}=i \zeta \frac{\partial}{\partial \zeta} \equiv \Xi \\
\tilde{X}_{(z)}^{R}=\frac{\eta^{-2}}{2(1+\kappa)}\left[(1+\kappa)^{2} \frac{\partial}{\partial z}+4 z^{* 2} \frac{\partial}{\partial z^{*}}-4 i z^{*} \frac{\partial}{\partial \theta}+2 i N z^{*} \Xi\right], \\
\tilde{X}_{\left(z^{*}\right)}^{R}=\frac{\eta^{2}}{2(1+\kappa)}\left[(1+\kappa)^{2} \frac{\partial}{\partial z^{*}}+4 z^{2} \frac{\partial}{\partial z}+4 i z \frac{\partial}{\partial \theta}-2 i N z \Xi\right], \\
\tilde{X}_{(\theta)}^{R}=\frac{\partial}{\partial \theta}, \\
\tilde{X}_{(\zeta)}^{R}=i \zeta \frac{\partial}{\partial \zeta} \equiv \Xi .
\end{gathered}
$$


The Lie algebra commutators are

$$
\begin{gathered}
{\left[\tilde{X}_{(z)}^{L}, \tilde{X}_{\left(z^{*}\right)}^{L}\right]=-2 i \tilde{X}_{(\theta)}^{L}-i N \Xi,} \\
{\left[\tilde{X}_{(\theta)}^{L}, \tilde{X}_{(z)}^{L}\right]=i \tilde{X}_{(z)}^{L}, \quad\left[\tilde{X}_{(\theta)}^{L}, \tilde{X}_{\left(z^{*}\right)}^{L}\right]=-i \tilde{X}_{\left(z^{*}\right)}^{L} .}
\end{gathered}
$$

From the last expressions (A.4) the characteristic subalgebra can be derived,

$$
\mathscr{G}_{\Theta}=\left\langle\tilde{X}_{(\eta)}^{L}\right\rangle,
$$

and the Full Polarization can be chosen as

$$
\mathscr{F} \mathscr{P}=\left\langle\tilde{X}_{(\eta)}^{L} \tilde{X}_{(z)}^{L}\right\rangle \text {. }
$$

The polarized wave functions, which satisfy

$$
\Xi \Psi=i \Psi, \quad \tilde{X}_{\mathscr{F} \mathscr{P}}^{L} \Psi=0,
$$

are linear combinations of the following basic functions $\left(\eta=e^{i \theta}\right)$ :

$$
|m\rangle \equiv \zeta(1+\kappa)^{-N / 2}\left[e^{-i \theta}(1+\kappa)^{-1} z^{*}\right]^{m}, \quad m=0,1,2,3, \ldots .
$$

The group representations (parametrized by the discrete index $N$ ) are given by the left translations acting on the wave functions,

$$
L_{\tilde{g}^{\prime}} \Psi(\tilde{\mathrm{g}})=\Psi\left(\tilde{\mathrm{g}}^{\prime} * \tilde{\mathrm{g}}\right) .
$$

The Lie algebra operators are the right-invariant vector fields acting on the wave functions as ordinary derivations [thus producing the infinitesimal version of (A.9)],

$$
\begin{aligned}
\tilde{X}_{(\theta)}^{R}|m\rangle & =-i m|m\rangle, \\
\tilde{X}_{(z)}^{R}|m\rangle & =-2(m+N)|m+1\rangle, \\
\tilde{X}_{\left(z^{*}\right)}^{R}|m\rangle & =\frac{1}{2} m|m-1\rangle .
\end{aligned}
$$

We get the usual $S L(2, \mathbb{R})$ action on $|m\rangle$ and Lie algebra commutators from the trivially redefined operators $\tilde{X}_{(\eta)}^{R^{\prime}}=\tilde{X}_{(\eta)}^{R}+N \Xi, \widetilde{X}_{(z)}^{R^{\prime}}=\widetilde{X}_{(z)}^{R}, \tilde{X}_{\left(z^{*}\right)}^{R^{\prime}}=\tilde{X}_{\left(z^{*}\right)}^{R}$.

It is very illustrative to realize, on the grounds of the present example, that even for (non-compact) finite-dimensional Lie groups one finds (polarized) wave functions not belonging to any orbit through the vacuum of the group. In fact, for negative $N$ only those wave functions with $m \leqq-N$ come from the vacuum. Of course this fact only happens (in finite dimensions) for non-unitary representations (negative $N$ ).

The scalar product of polarized wave functions out of which unitary representations can be found is given by

$$
\left\langle\psi^{\prime}, \psi\right\rangle=\int_{S L(2, \mathbb{R})} \theta^{L_{(z)}} \wedge \theta^{L_{\left(z^{\star}\right)}} \wedge \theta^{L_{(\eta)}} \psi^{\prime *} \psi,
$$

where $\vartheta^{L}$ are (the non-vertical) components of the canonical left-invariant 1 -form,

$$
\begin{aligned}
\theta^{L_{(z)}} & =\frac{1+\kappa}{\kappa} d z-\frac{4 z^{2}}{\kappa(1+\kappa)} d z^{*}+2 i z d \theta \\
\theta^{L_{\left(z^{*}\right)}} & =\frac{1+\kappa}{\kappa} d z^{*}-\frac{4 z^{* 2}}{\kappa(1+\kappa)} d z-2 i z^{*} d \theta \\
\theta^{L_{(\eta)}} & =\frac{-i z^{*}}{1+\kappa} d z+\frac{i z}{1+\kappa} d z^{*}+\frac{1}{2} \kappa d \theta
\end{aligned}
$$


The invariant volume is

$$
\mu \equiv \theta^{L_{(z)}} \wedge \theta^{L_{\left(z^{*}\right)}} \wedge \theta^{L_{(\eta)}}=\frac{2}{\kappa} d z \wedge d z^{*} \wedge d \theta
$$

and the scalar product between basic states $|m\rangle$ is

$$
\langle m \mid n\rangle=8 \pi \delta_{m, n} \int d z \wedge d z^{*} \frac{|z|^{2 m}}{\kappa(1+\kappa)^{N+2 m}} .
$$

A group representation will in principle be unitary $\left(\tilde{X}^{R^{\dagger}}=-\tilde{X}^{R}\right)$ if the integral is finite. Among the values of the parameter $N$ characterizing the representations only $N \geqq 2$ make the wave functions square-integrable with respect to the scalar product (A.14). Nevertheless, from the strict Lie algebra point of view, for which $N$ need not be an integer, the operators (A.10) are anti-hermitian for just $N>1$. In addition, the scalar product (A.14) can be continued to the value $N=1$ which defines a group representation (the Mock representation):

$$
\langle m \mid n\rangle_{N=1}=\lim _{N \rightarrow 1^{+}} \frac{\langle m \mid n\rangle_{N}}{\langle 0 \mid 0\rangle_{N}} .
$$

So then, and among the class of representations here studied, those representations are unitary for which $N=1,2,3, \ldots$. The rest of the (unitary) group representations can be constructed within the same framework taking the other two ( $\mathbb{R}$-)fibrations of $S L(2, \mathbb{R})$, one over the 1-sheeted hyperboloid and another over the light-cone.

Acknowledgements. One of us (V.A.) is grateful to C. J. Isham for his hospitality at Imperial College.

\section{References}

1. Souriau, J.M.: Structure des Systemes dynamiques. Paris: Dunod 1970; Kostant, B.: Quantization and Unitary Representation. In: Lecture Notes in Mathematics, Vol. 179, Berlin, Heidelberg, New York: Springer 1970; Kirillov, A.A.: Elements of the Theory of Representations. Berlin, Heidelberg, New York: Springer 1975

2. Witten, E.: Commun. Math. Phys. 114, 1 (1988); Segal, G.: Commun. Math. Phys. 80, 307 (1981)

3. Ne'eman, Y., Regge, T.: Gauge theory of gravity and supergravity on a group manifold. Riv. Nuovo Cim. 1, fasc. 5 (1978)

4. Bayen, F., Flato, M., Fronsdal, C., Lichnerowicz, A., Sternheiner, D.: Deformation theory and quantization. I: deformation of symplectic structures. Ann. Phys. 111, 61 (1978); Deformation theory and quantization. II: physical applications. Ann. Phys. 111, 111 (1978)

5. Aldaya, V., de Azcárraga, J.A.: J. Math. Phys. 23, 1297 (1982); Fortschr. Phys. 35, 437 (1987); Proceeding of the Conference on "Constraints Theory and Relativistic Dynamics," Firence 1986, Singapore: World Scientific, Longhi, G., Lusana, L. (eds.)

6. Aldaya, V., de Azcárraga, J.A.: Ann. Phys. (NY) 165, 484 (1985)

7. Isham, C.J., Kakas, A.C.: Class. Quant. Grav. 1, 621 (1984); 1, 633 (1984); Isham, C.J., Linden, N.: Class. Quant. Grav. 5, 71 (1988)

8. Bakas, I., Kakas, A.C.: J. Phys. A20, 3713 (1987)

9. Drinfel'd, V.G.: Sov. Math. Dokl. 27, 68 (1983); 32, 254 (1985)

10. Aldaya, V., Navarro-Salas, J.: Commun. Math. Phys. 113, 375 (1987)

11. Aldaya, V., Navarro-Salas, J.: Lett. Math. 16, 117 (1988) 
12. Aldaya, V., Navarro-Salas, J., Ramírez, A.: Algebraic quantization on a group and nonabelian constraints. Commun. Math. Phys. 121, 541-556 (1989)

13. Bargmann, V.: Ann. Math. 59, 1 (1954)

14. Aldaya, V., de Azcárraga, J.A., Bisquert, J., Cervero, J.M.: J. Phys. A (to appear)

15. Lang, S.: $S L_{2}(\mathbb{R})$, Reading, MA: Addison Wesley 1975

16. Kac, V.G.: Lecture Notes in Phys., Vol. 94, 441. Berlin, Heidelberg, New York: Springer 1979

17. Kobayashi, S., Nomizu, K.: Foundations of differential geometry, Vol. I, New York: Interscience 1963

18. Serre, J.P.: Lie algebras and Lie groups. New York: Benjamin 1965. Formal group laws for infinite-dimensional groups as well as right- and left-invariant vector fields are considered in $\mathbf{J}$. Milnor: In: Relativity, groups and topology II, Les Houches 1983

19. Bowick, M.J., Rajeev, S.G.: Phys. Rev. Lett. 58, 535 (1987); Nucl. Phys. 293B, 348 (1987); CTP 1494 (1987)

20. Pressly, A., Segal, G.: Loop groups. Oxford: Clarendon Press 1986

21. Friedan, D., Qiu, Z., Shenker, S.: Phys. Rev. Lett. 52, 1575 (1984)

22. Gotay, M.J.: Monatssch. Math. 103, 27 (1987)

23. Aldaya, V., Loll, R., Navarro-Salas, J.: Phys. Lett. B 225, 340 (1989)

Communicated by A. Jaffe

Received August 1, 1988; in revised form May 8, 1989 
